Supporting Information for Publication:

Strain-Tunable Carbon Nanothread-Derived Membranes for Water Desalination

Pedro G. Demingos, Rafaela A. Pagnussatti, André R. Muniz*

Department of Chemical Engineering, Universidade Federal do Rio Grande do Sul, Rua Luiz Englert s/n, 90040-040, Porto Alegre, RS, Brazil

*E-mail: amuniz@enq.ufrgs.br 
1. Atomic charges and Lennard-Jones parameters

\begin{tabular}{ccccc}
\hline Atom & Description & $\begin{array}{c}\text { LJ Sigma } \\
(\AA)\end{array}$ & $\begin{array}{c}\text { LJ Epsilon (Kcal// } \\
\text { mol) }\end{array}$ & $\begin{array}{c}\text { Charge } \\
\text { (parts of } \boldsymbol{e} \text { ) }\end{array}$ \\
\hline $\mathrm{Ch}$ & C bonded to 1 H & 3.56359 & 0.032 & -0.09 \\
$\mathrm{Hc}$ & H bonded to C & 2.38761 & 0.045 & 0.09 \\
$\mathrm{Cc}$ & $\mathrm{C}$ bonded to only C & 3.56359 & 0.032 & 0 \\
$\mathrm{Ow}$ & water O & 3.15057 & 0.1521 & -0.834 \\
$\mathrm{Hw}$ & water H & 0.4 & 0.046 & 0.417 \\
$\mathrm{Cl}$ & chloride anion & 4.47766 & 0.0356 & -1 \\
$\mathrm{Na}$ & sodium cation & 2.43928 & 0.0874 & 1 \\
\hline
\end{tabular}

Table S1. Charges and Lennard-Jones parameters used for the simulations. Charges are given in terms of the fundamental charge. 


\section{Additional results}

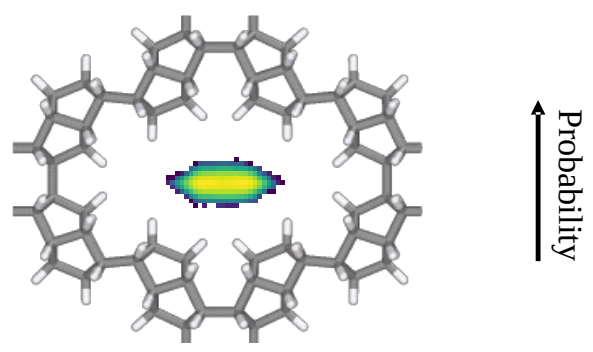

Figure S1. Structure of CNM-2 along with water density map computed during filtration.

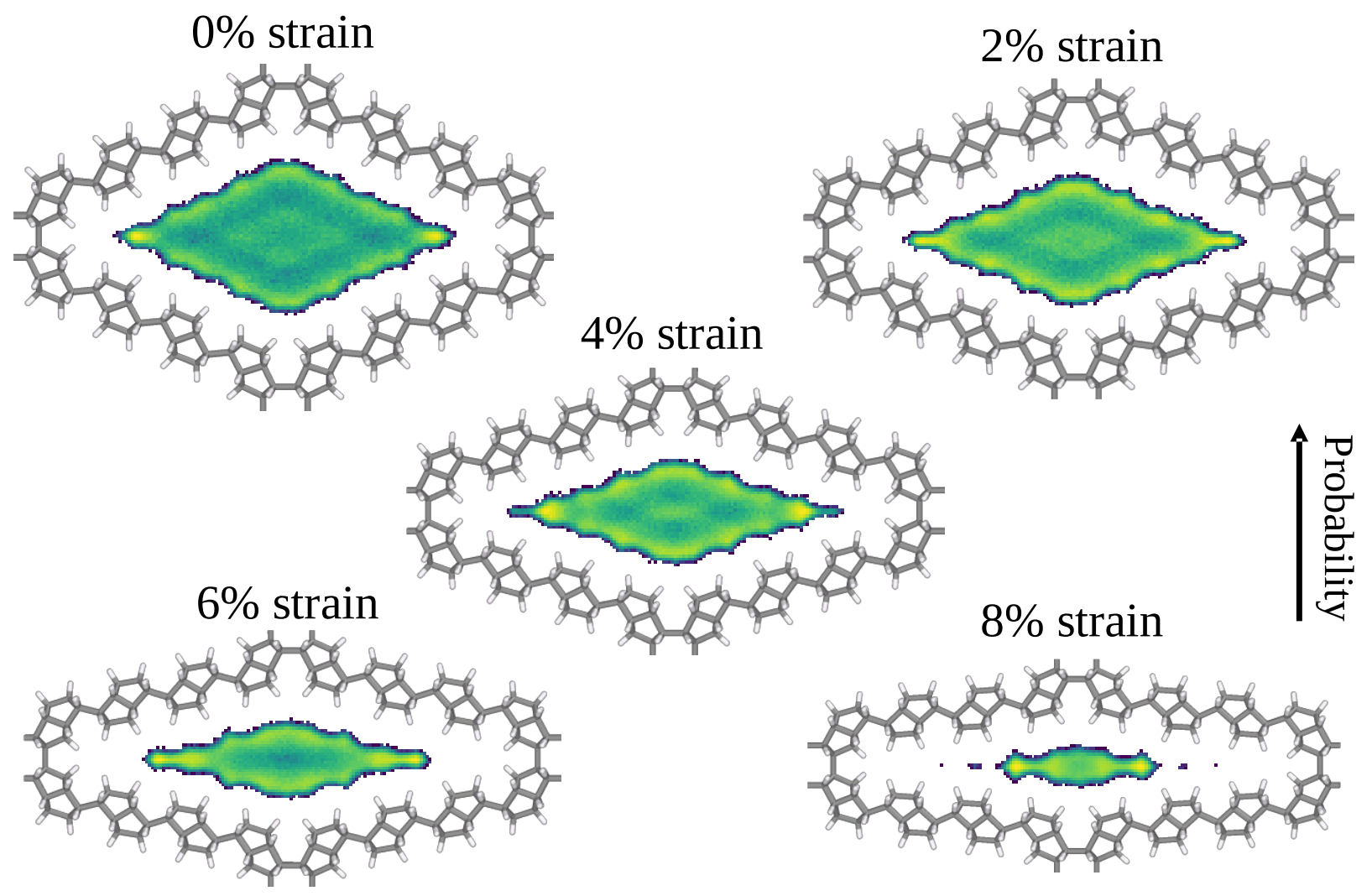

Figure S2. Structure of CNM-4 at different applied strains, along with water density maps computed during filtration. 

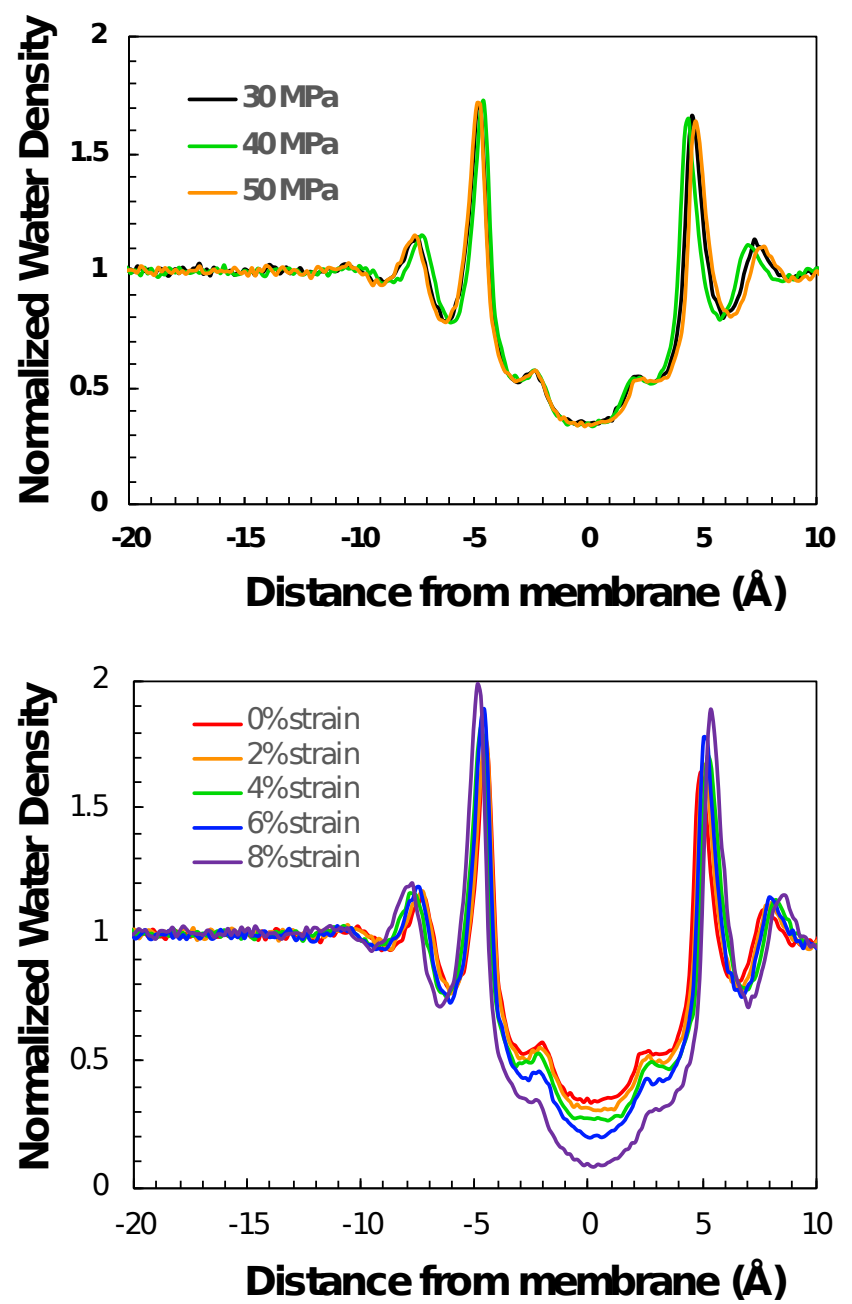

Figure S3. Water density profile for CNM-4 (top) at zero strain and different pressures and (bottom) at $50 \mathrm{MPa}$ and different strains. Water density is normalized by the bulk water density and shown along the simulation axis that is perpendicular to the membrane plane (the origin of the axis, $x=0$, coincides with the center of the membrane).

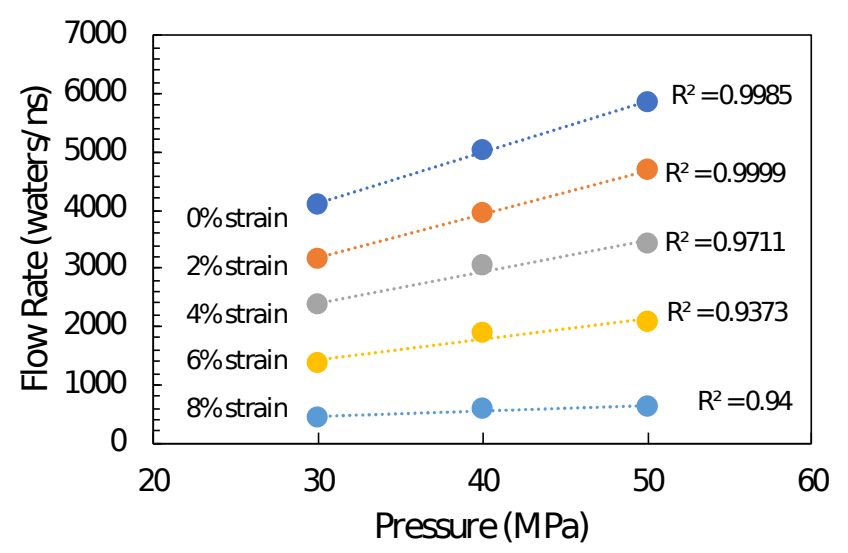

Figure S4. Linear relation between water flow and applied pressure for CNM-3 at different externally applied uniaxial tensile strain. 
(a)

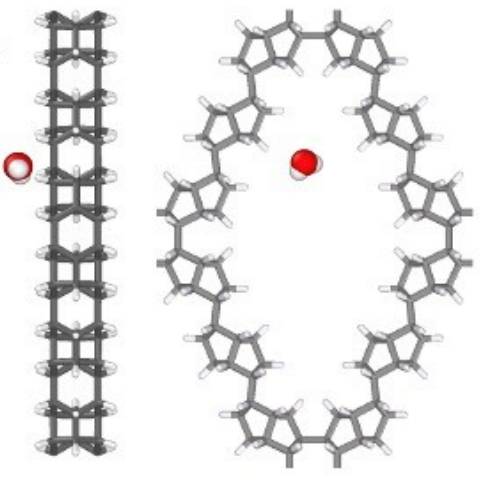

(b)

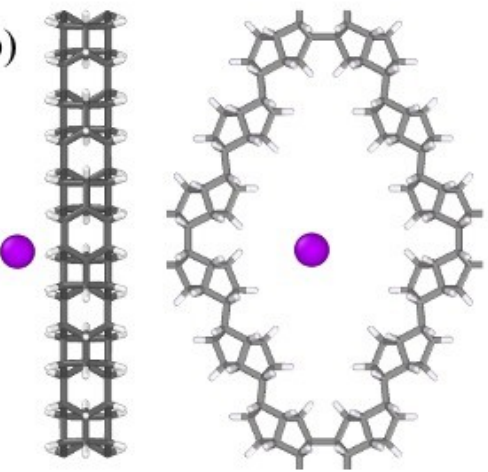

(c)

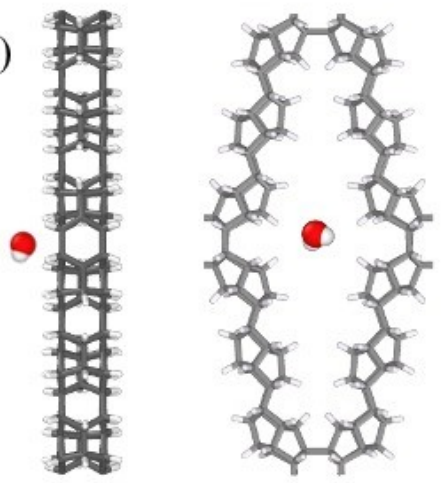

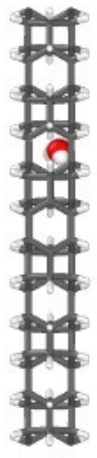
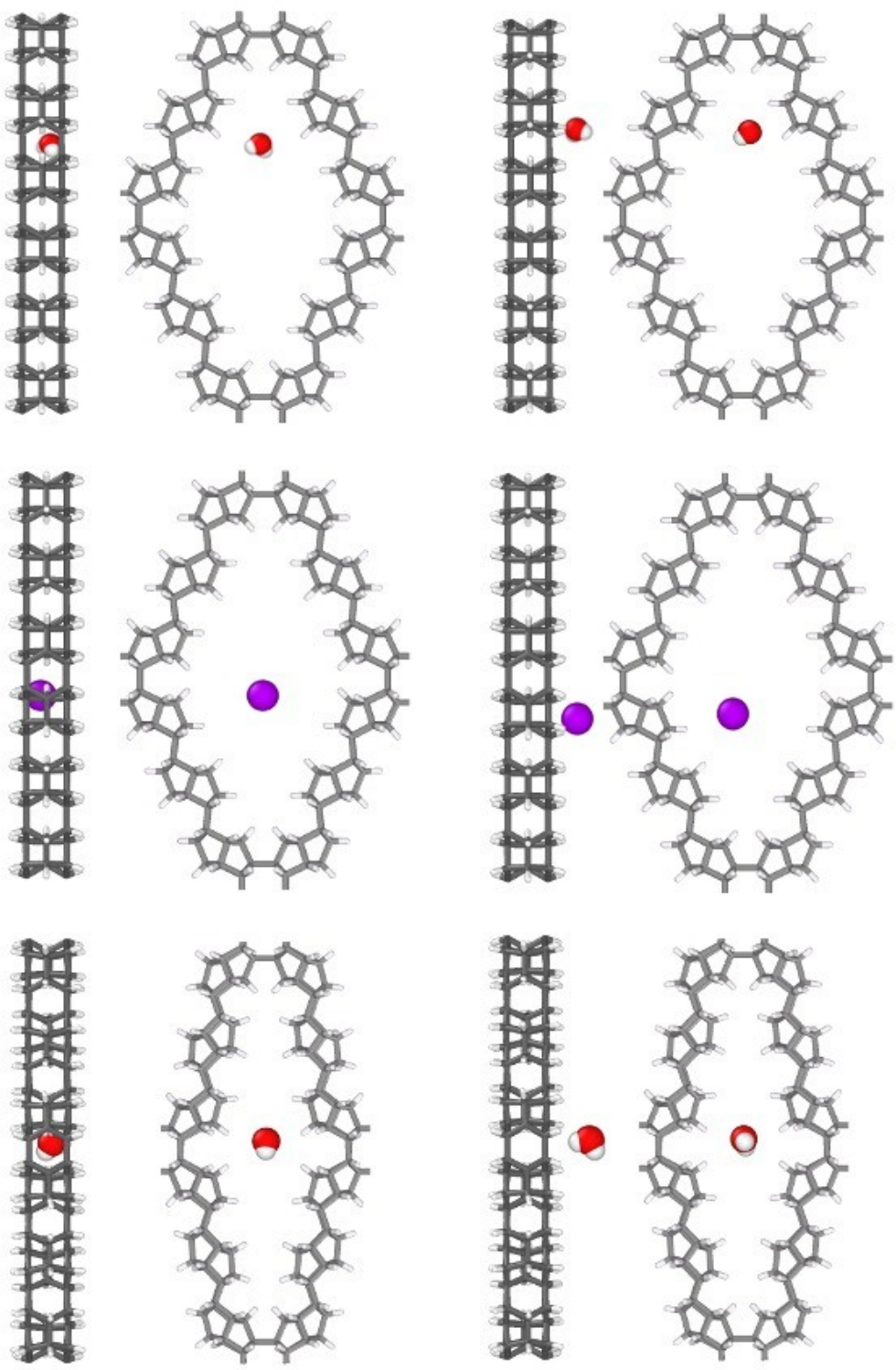

Figure S5. Snapshots of the simulations showing the passage of species across the membranes: before (left), during (middle), and after (right). (a) Water through CNM-3 at 0\% strain, (b) Chloride ion through CNM-3 at 0\% strain, (c) Water through CNM-3 at 8\% strain. 


\section{Data fitting and computation of water fluxes}

Regarding the data fitting procedure to compute water fluxes: Figure 2 of the manuscript shows that within 5 ns, the dependence of the number of water molecules filtered with time reaches a linear regime (equivalent to a constant flow rate). In fact, similarly to what was reported by Cohen-Tanugi [8], at least one of our simulations (CNM-4 at 0\% strain) shows a clearly linear regime within the first 1 ns of filtration, strongly suggesting that simulation time is not an issue to capture a linear flux regime. Moreover, Figure S6 shows the linear regression for the number of filtered water molecules using data for a faster and a slower process (from 0 up to $1 \mathrm{~ns}$ and to $5 \mathrm{~ns}$, respectively), and both curves allow an excellent fit. We also note that these curves are shown after a suitable period of previous equilibration before allowing the molecules to cross the membrane as mentioned in the paper, which is important to guarantee the linear regime that is observed in the initial stages [22]. Again, as seen in the Figure, the absolute number of water molecules used in our simulations is considerably higher than those employed in similar works.

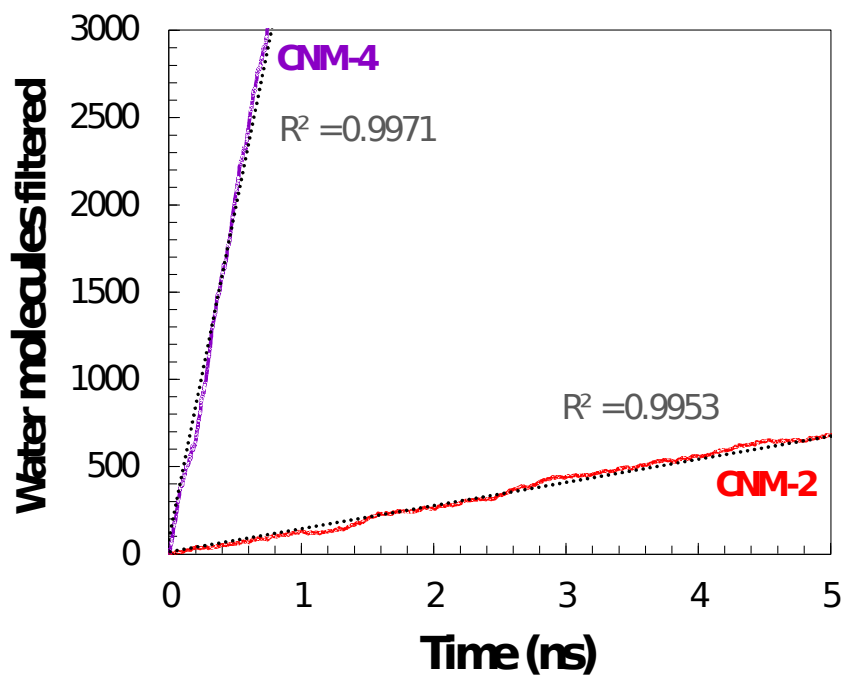

Figure S6. Number of filtered water molecules as function of time in the linear regime of water flux, along with their corresponding linear regression results, for selected simulations (CNM-2 and CNM-4 at $30 \mathrm{MPa}$ and $0 \%$ applied strain). 